\section{L'ankyloglossie et l'allaitement}

\author{
Anne Rowan-Legg MD, Société canadienne de pédiatrie, \\ comité de la pédiatrie communautaire
}

\section{HISTORIQUE}

L'ankyloglossie est une anomalie congénitale qu'on observe chez les nouveau-nés et les enfants et qui se caractérise par un frein lingual anormalement court. Un frein trop court peut restreindre la mobilité de la langue à divers degrés.

Les associations entre l'ankyloglossie, les problèmes de lactation, les troubles d'élocution et d'autres troubles oromoteurs (p. ex., problèmes de déglutition ou de léchage) ne sont pas univoques et sont une source de controverse constante au sein du corps médical.(1-3) Un sondage auprès d'otorhinolaryngologistes, de pédiatres, d'orthophonistes et de conseillères en lactation a révélé des écarts importants au sein de ces groupes et entre eux quant à leur façon d'aborder l'ankyloglossie et à leurs convictions sur son association avec les problèmes d'alimentation, les troubles d'élocution et les problèmes sociaux.(1) Les dentistes sont tout aussi divisés sur le sujet.(4)

Puisque les bienfaits de l'allaitement (que la Société canadienne de pédiatrie soutient entièrement) sont réitérés, les pressions sont plus fortes que jamais pour diagnostiquer l'ankyloglossie comme cause d'échec de l'allaitement, accroissant ainsi la demande de frénotomie.

Le présent document de principes porte tout particulièrement sur les preuves entourant l'association entre l'ankyloglossie et les problèmes d'allaitement chez les nourrissons et sur l'efficacité de la frénotomie en cas d'ankyloglossie et de problèmes d'allaitement. Il remplace celui que la Société canadienne de pédiatrie a révisé en 2011.(5) Plusieurs études ont été publiées depuis.

\section{DÉFINITION}

Il n'existe ni définition universelle de l'ankyloglossie ni critères objectifs pratiques pour la diagnostiquer. D'ordinaire, les définitions reposent sur les caractéristiques anatomiques du frein lingual (le degré de fusion entre la langue et le plancher de la bouche de l'enfant) ou sur une atteinte fonctionnelle (une incapacité de faire sortir la langue au-delà du bord incisif de la gencive inférieure et d'autres signes d'entrave à la mobilité de la langue).(1,6-9) Selon un système de classification, l'ankyloglossie de types I et II se caractérise par une insertion au bout de la langue (type I) ou légèrement auparavant (type II), tandis que l'ankyloglossie postérieure se caractérise par l'épaississement du frein (type III) ou par un frein sous-muqueux (un monticule lingual large et plat) qui restreint le mouvement à la base de la langue (type IV).(10) Ces définitions sont peu utilisées dans les publications et rarement en milieu clinique. Hazelbaker(11) a mis au point un outil descriptif d'évaluation de la fonction du frein lingual. Cependant, il est long et complexe et n'a pas été validé de manière contrôlée.(12) Les

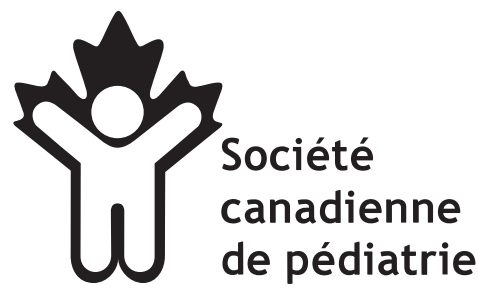

English on page 209

Résumé en page 209 critères utilisés pour diagnostiquer l'ankyloglossie sont très variables, et il n'existe pas de norme acceptée. L'absence de définition universelle contribue également à alimenter la controverse à l'égard de cette affection et de sa signification clinique.

\section{L'ÉTIOLOGIE}

Au début du développement fœetal, la langue est fusionnée au plancher de la bouche. La mort et la résorption des cellules libèrent la langue, et le frein demeure le seul vestige de cette fusion initiale. D'ordinaire, le frein lingual se résorbe quelque peu pendant le processus naturel de la croissance et du développement, lorsque croissent les crêtes alvéolaires et que commence l'éruption des dents.(2) Ce processus se produit entre l'âge de six mois et de cinq ans. L'ankyloglossie peut être classée d'après le degré de fusion qui persiste entre la langue et le plancher de la bouche.(2)

Il peut y avoir une prédisposition génétique à l'ankyloglossie. (13) D'ordinaire, cette anomalie congénitale ne s'associe pas à d'autres manifestations.

\section{LA PRÉVALENCE}

La prévalence déclarée d'ankyloglossie chez les nourrissons varie dans les publications, ce qui reflète l'absence de définition uniforme. Les évaluations varient entre $4,2 \%$ et $10,7 \%$ chez les nouveau-nés.(7-9)

\section{LA PHYSIOPATHOLOGIE}

De nombreux rapports isolés font état du rôle du frein lingual court dans les problèmes d'allaitement, qu'ils lient à une mauvaise prise du sein, à des douleurs et des traumatismes mammaires chez la mère, à une prise de poids sous-optimale par le nourrisson, au refus de prendre le sein et à une insuffisance de lait maternel causée par l'éjection médiocre du lait.(14-17) Pour bien téter, le nourrisson doit s'accrocher à l'aréole de la mère au moyen de la crête supérieure des gencives, des boules graisseuses de Bichat et de la langue. La tétée s'amorce par l'avancement de la mâchoire et de la langue. La langue assure une meilleure étanchéité de la tétée, mais joue un rôle minimal. La crête antérieure de la langue s'amincit et se bombe pour entreprendre une ondulation péristaltique vers la gorge. La mâchoire inférieure extrait simultanément le lait des canaux lactifères.(18) De toute évidence, les mouvements de la langue doivent être extrêmement entravés pour nuire à la tétée et à la déglutition.(2) Il semble également que les seins, les mamelons ou l'éjection du lait de certaines mères présentent des caractéristiques particulières qui leur permettent d'allaiter sans problème un bébé ayant une ankyloglossie.(16)

D’après des études échographiques, le mécanisme d'alimentation déficiente des nourrissons ayant une ankyloglossie est causé par un

Correspondance : Société canadienne de pédiatrie, 2305, boulevard St Laurent, Ottawa (Ontario) K1G 4J8, courriel : info@cps.ca, site Web : www.cps.ca 
mouvement restreint de la langue qui peut provoquer une douleur ou un traumatisme du mamelon, une éjection médiocre du lait et l'incapacité de s'accrocher au sein. La frénotomie semble rétablir des mouvements de tétée qui ressemblent davantage à ceux des nourrissons qui n'ont pas d'ankyloglossie.(19)

\section{L'ANKYLOGLOSSIE ET LES PROBLÈMES D'ALLAITEMENT}

Plusieurs études ont porté sur l'association de l'ankyloglossie aux problèmes d'allaitement. Une étude a comparé le taux d'ankyloglossie chez des nourrissons qui fréquentaient une clinique ambulatoire sur les problèmes d'allaitement à la population générale de nouveau-nés à terme et en santé et a conclu en une plus forte incidence d'ankyloglossie chez les nourrissons ayant des problèmes d'allaitement (12,8\% par rapport à 3,2\%).(8)

Une autre étude a porté sur une cohorte de 201 nouveau-nés ayant une ankyloglossie et rendu compte d'une forte incidence de troubles de tétée (44\%), mais n'a pas établi de lien entre la gravité de l'ankyloglossie et les problèmes d'allaitement. Cette étude a également démontré que $56 \%$ des nourrissons ayant une ankyloglossie peuvent se nourrir correctement.(6)

Un essai prospectif a révélé une plus forte incidence de troubles de prise du sein (19\% par rapport à $0 \%$ ) et de problèmes d'allaitement (25\% par rapport à $3 \%$ ) dans un groupe de 36 nouveau-nés ayant une ankyloglossie comparativement à un groupe témoin de nouveau-nés sans ankyloglossie. Trente des 36 nourrissons ayant une ankyloglossie (83\%) ont été allaités sans problème pendant la période de l'étude, par rapport à 33 des 36 nourrissons témoins $(92 \%)(P=0,29)$. La durée de l'allaitement était similaire dans les deux groupes.(9) Par ailleurs, cette étude n'a permis de constater aucune différence significative du degré d'ankyloglossie (modéré ou léger) ou de l'épaisseur du frein chez les nourrissons ayant des problèmes d'allaitement.(9)

\section{LA PRISE EN CHARGE DE L'ANKYLOGLOSSIE}

En général, l'ankyloglossie est traitée avec prudence. Elle n'exige pas d'intervention à part de l'information aux parents, un soutien à l'allaitement et des paroles rassurantes. Si l'ankyloglossie s'associe à d'importants problèmes d'allaitement, certaines données indiquent que la frénotomie peut améliorer la situation. La controverse demeure quant aux ankyloglossies à corriger par voie chirurgicale et celles qui peuvent être simplement observées.

Plusieurs études, y compris de récentes études aléatoires et contrôlées, ont visé à évaluer l'efficacité de la frénotomie pour corriger une ankyloglossie en cas de problèmes d'allaitement (tableau 1). $(6,8,20-27)$.

Ces études aléatoires et contrôlées et ces essais prospectifs comportent d'importantes limites, y compris les suivantes :

- La variabilité et la définition imprécise du diagnostic d'ankyloglossie, qui s'associent à des critères d'inclusion flous

- La petite taille des échantillons cliniques de ces études

- Dans la plupart des études, le croisement de presque tout le groupe témoin dans le groupe subissant la frénotomie, ce qui empêche la juste évaluation des résultats et en complique l'interprétation

- Des mesures objectives des résultats souvent limitées et fondées sur l'observation d'un seul boire;

- L'absence de données démographiques (mères d'un premier enfant ou mères expérimentées ayant déjà plusieurs enfants);

- Des résultats parfois mal définis (p. ex., « amélioration de l'alimentation »)
Il est très difficile d'assurer l'insu des observateurs et des mères dans de telles études. En effet, dans une étude, 100 \% des mères expérimentées ont bien identifié la correction de l'ankyloglossie de leur nourrisson effectuée à leur insu.(22) Il faut également évaluer avec prudence la capacité d'une nouvelle mère à répondre objectivement à des questions sur l'amélioration de l'allaitement lorsque son nourrisson vient de subir une intervention à laquelle elle a consenti.

Étonnamment, très peu de publications décrivent la courbe d'apprentissage « normale » de l'allaitement chez la mère et son nourrisson. En raison de cette lacune et de la rareté du maintien des groupes témoins pendant les études, il est difficile d'établir si les problèmes d'allaitement se seraient atténués au fil des semaines et grâce à une prise en charge prudente (l'évolution naturelle de la situation).

Outre les études décrites, plusieurs autres études prospectives de cohorte ont révélé une association entre l'ankyloglossie et les problèmes d'allaitement,(28) de même que les avantages de la frénotomie chez les nourrissons ayant une ankyloglossie qui éprouvaient des problèmes d'allaitement.(10,29-32) Ces études partagent toutefois certaines des limites exposées ci-dessus.

Ainsi, même si plusieurs essais aléatoires, certaines études de cohorte et quelques études transversales prospectives ont démontré une certaine efficacité de la frénotomie chez les nouveau-nés éprouvant des problèmes d'allaitement causés par l'ankyloglossie, ces études comportaient toutes d'importantes limites.

\section{LA FRÉNOTOMIE}

S'il est jugé nécessaire de corriger une ankyloglossie, il faut orienter le nourrisson vers un otorhinolaryngologiste ou un médecin qui a l'habitude de cette intervention. Il faut prévoir une analgésie suffisante. Malheureusement, les publications portant sur l'analgésie efficace pour effectuer une frénotomie sont rares. L'utilisation d'acétaminophène, de lidocaïne et de sucrose est citée dans les rapports de cas, mais aucun de ces produits n'a été étudié systématiquement. Un essai aléatoire et contrôlé a démontré que l'effet de la benzocaïne était équivalent à celui d'un placebo.(33) La libération d'une ankyloglossie semble être une intervention mineure, mais elle peut provoquer des complications, telles que des saignements, une infection ou une lésion du canal de Wharton. D'après les quelques publications sur le sujet, l'incidence de complications mineures semble rare.(22)

Une simple incision du frein (la frénotomie) est la principale intervention effectuée pour traiter une ankyloglossie partielle. Elle s'associe au risque que la cicatrisation postopératoire limite davantage le mouvement de la langue, ce qui nécessiterait une nouvelle opération. L'excision accompagnée d'un allongement de la surface ventrale de la langue et la frénuloplastie sont des interventions plus complexes. Toutes deux favorisent une cicatrisation postopératoire moins importante, mais exposent le nourrisson aux risques de l'anesthésie générale. $(33,34)$

Des cliniques privées spécialisées procèdent désormais à la frénotomie par ablation au laser, mais on possède peu de données sur l'innocuité ou l'efficacité de cette intervention.

\section{CONCLUSION}

L'ankyloglossie est relativement courante chez les nouveau-nés. La plupart du temps, il s'agit d'une caractéristique anatomique sans conséquences importantes pour les nourrissons touchés. D'après les données probantes à jour, la plupart des nouveau-nés touchés seraient en mesure de téter sans problème.

D'après les données probantes, on ne peut pas recommander la frénotomie chez tous les nourrissons ayant une ankyloglossie. Il n'y a aucun lien absolu entre l'ankyloglossie et les problèmes d'allaitement. 
TABLEAU 1

Études sur l'efficacité de la frénotomie chez les nourrissons ayant une ankyloglossie et des problèmes d'allaitement

\begin{tabular}{|c|c|c|c|}
\hline $\begin{array}{l}\text { Auteurs } \\
\text { (référence), } \\
\text { année }\end{array}$ & Type d'étude & $\begin{array}{c}\text { Population à } \\
\text { l'étude }\end{array}$ & $\begin{array}{c}\text { Définition } \\
\text { d'ankyloglossie }\end{array}$ \\
\hline $\begin{array}{l}\text { Hogan et coll. } \\
(6), 2005\end{array}$ & $\begin{array}{l}\text { Essai } \\
\text { aléatoire et } \\
\text { contrôlé }\end{array}$ & $\begin{array}{l}\text { 57 nourrissons } \\
\text { ayant une } \\
\text { ankyloglossie et } \\
\text { des problèmes } \\
\text { pour se nourrir } \\
\text { (au sein ou au } \\
\text { biberon) } \\
\text { Âge moyen au } \\
\text { moment de la } \\
\text { répartition aléa- } \\
\text { toire : } 20 \text { jours }\end{array}$ & $\begin{array}{l}\text { Pourcentage } \\
\text { d'ankyloglossies } \\
\text { évaluées à l'œil, } \\
\text { se situant entre } \\
100 \% \text { (jusqu'au } \\
\text { bout de la } \\
\text { langue) et } 25 \%\end{array}$ \\
\hline $\begin{array}{l}\text { Dollberg et coll. } \\
\text { (20), } 2006\end{array}$ & $\begin{array}{l}\text { Étude } \\
\text { aléatoire } \\
\text { prospective } \\
\text { à simple } \\
\text { insu }\end{array}$ & $\begin{array}{l}25 \text { nourrissons } \\
\text { (de } 1 \text { à } \\
21 \text { jours) ayant } \\
\text { une } \\
\text { ankyloglossie } \\
\text { et dont la mère } \\
\text { éprouvait des } \\
\text { douleurs aux } \\
\text { mamelons }\end{array}$ & $\begin{array}{l}\text { « Incapacité du } \\
\text { nourrisson à } \\
\text { faire sortir la } \\
\text { langue au-delà } \\
\text { de la gencive } \\
\text { inférieure parce } \\
\text { qu'elle était } \\
\text { attachée au } \\
\text { plancher de la } \\
\text { bouche par un } \\
\text { frein serré, et } \\
\text { que la langue } \\
\text { prenait la forme } \\
\text { d'un cœur } \\
\text { lorsqu'elle était } \\
\text { soulevée. » }\end{array}$ \\
\hline $\begin{array}{c}\text { Berry et coll. } \\
(22), 2012\end{array}$ & $\begin{array}{l}\text { Essai } \\
\text { aléatoire et } \\
\text { contrôlé à } \\
\text { double insu }\end{array}$ & $\begin{array}{l}60 \text { nourrissons } \\
\text { allaités (âge } \\
\text { moyen de } \\
32 \text { jours) ayant } \\
\text { des problèmes } \\
\text { d'allaitement } \\
\text { (définis par des } \\
\text { problèmes de } \\
\text { prise du sein, } \\
\text { des douleurs } \\
\text { ou des } \\
\text { traumatismes } \\
\text { aux mamelons, } \\
\text { ou une tétée } \\
\text { inefficace) et } \\
\text { une } \\
\text { ankyloglossie }\end{array}$ & $\begin{array}{l}\text { «Présence } \\
\text { d'ankyloglossie } \\
\text { » }\end{array}$ \\
\hline
\end{tabular}

Méthodologie

Affectés au hasard

entre une

frénotomie ou un

groupe témoin

(conseil)

En l'absence

d'amélioration

pendant 48 heures

dans le groupe

témoin, offre de

frénotomie

Affectés au hasard Après la première intervention

à l'une des deux (frénotomie ou placebo),

séquences mesure du score standardisé de

suivantes : i) une LATCH(21) et évaluation

frénotomie et

l'allaitement, une

échelonnée de la douleur par la

mère (de 1 à 10)

intervention

placebo et

l'allaitement

(14 nourrissons)

ii) une

intervention

placebo et

l'allaitement, une

frénotomie et

l'allaitement

(11 nourrissons)

Affectés au hasard Avant l'intervention: Score de entre une

frénotomie

immédiate

(30 nourrissons)

ou l'absence de

frénotomie

(30 nourrissons)

LATCH et outil d'évaluation de

l'allaitement du nourrisson

(IBFAT) (23) et score de

douleur de la mère (de 1 à 10$)$

pendant la tétée observée

Après l'intervention (premier

boire déclaré être à l'insu) :

Évaluation objective comme ci-dessus

Évaluation subjective par la mère et score de la douleur (de 1 à 10)

Appel téléphonique au bout d'1 jour et de 3 mois pour connaître les modifications subjectives des tétées, des complications et les taux d'allaitement

Emond et coll. Essai aléa- 107 nourrissons (24), 2014 toire et con- à terme (âge trôlé médian de

11 jours) ayant

une

ankyloglossie

légère à

modérée et des

problèmes

d'allaitement

(définis comme

un score de

LATCH $\leq 8$ )
Outil d'évaluation Affectés au hasard Résultat primaire : score de de Hazelbaker entre une pour le score de la fonction du frein lingual (HATLFF) de 6 à

12

frénotomie immédiate ou des soins standards

LATCH à 5 jours

Résultats secondaires : score de LATCH à 8 semaines et IBFAT à 5 jours, puis à 8 semaines

Questionnaire court du score d'auto-efficacité de l'allaitement (BSES-SF)(25) et échelle de la douleur (de 1 à 10) à 5 jours, puis à 8 semaines et poids du nourrisson à 8 semaines
Les mères de 21 des 27 nourrissons (78 \%) du groupe ayant subi la frénotomie ont déclaré une amélioration subjective après l'intervention, par rapport à 14 des 30 nourrissons (47\%) du groupe témoin

L'observateur objectif a déclaré que l'amélioration de la tétée n'était pas statistiquement significative (50\% d'amélioration dans le groupe ayant subi une frénotomie et $40 \%$ dans le groupe témoin)

30 nourrissons dans le groupe n'ayant pas subi la frénotomie ont ensuite subi l'intervention

3 mois après la frénotomie, $56 \%$ (33 sur 59 ) ont déclaré une pleine résolution, et $8 \%$ (5 sur 59 ), aucune amélioration

Le taux d'allaitement s'élevait à $51 \%$ au suivi de 3 mois

À 5 jours de vie, le score de HATLFF était plus élevé dans le groupe ayant subi la frénotomie, mais l'IBFAT et le score de LATCH ou le BSES-SF et la douleur n'avaient pas changé

35 des 53 nourrissons du groupe témoin se sont fait offrir une frénotomie au bout de 5 jours

Aucune différence entre les groupes dans les évaluations de l'allaitement ou dans le poids du nourrisson au bout de 8 semaines

Taux d'allaitement de $80 \%$ dans les 2 groupes au bout de 8 semaines 
TABLEAU 1 (suite)

Études sur l'efficacité de la frénotomie chez les nourrissons ayant une ankyloglossie et des problèmes d'allaitement

\begin{tabular}{|c|c|c|c|c|c|c|}
\hline $\begin{array}{l}\text { Auteurs } \\
\text { (référence), } \\
\text { année }\end{array}$ & Type d'étude & $\begin{array}{l}\text { Population à } \\
\text { l'étude }\end{array}$ & $\begin{array}{c}\text { Définition } \\
\text { d'ankyloglossie }\end{array}$ & Méthodologie & Mesures de résultats & Résultats \\
\hline $\begin{array}{l}\text { Ballard et coll. } \\
(8), 2002\end{array}$ & $\begin{array}{l}\text { Étude de } \\
\text { cohorte }\end{array}$ & $\begin{array}{l}127 \text { nourrissons } \\
\text { ayant une } \\
\text { ankyloglossie } \\
\text { recrutés (sur } \\
3036 \\
\text { nourrissons à } \\
\text { terme } \\
\text { hospitalisés ou } \\
\text { non) }\end{array}$ & $\begin{array}{l}\text { Score de fonction } \\
\text { de HATLFF } \\
>11 \text { sur } 14 \text { en } \\
\text { présence d'un } \\
\text { score d'apparence } \\
\text { de moins de } 8 \text { sur } \\
10\end{array}$ & $\begin{array}{l}123 \text { nourrissons } \\
\text { ont subi une } \\
\text { frénotomie } \\
\text { (4 mères ont } \\
\text { refusé } \\
\text { l'intervention) }\end{array}$ & $\begin{array}{l}\text { Prise du sein non mesurée de } \\
\text { manière quantitative; mesure } \\
\text { subjective par les évaluateurs et } \\
\text { la mère } \\
\text { Score de douleur des mamelons } \\
\text { par la mère (de } 1 \text { à 10) }\end{array}$ & $\begin{array}{l}\text { Ankyloglossie considérée comme } \\
\text { responsable de } 35 \text { des } \\
\text { t } 273 \text { problèmes d'allaitement (12,8 \%) } \\
\text { vus en consultations externes } \\
\text { Scores de HATLFF moyens } \\
\text { similaires en présence de } \\
\text { caractéristiques cliniques de } \\
\text { mauvaise prise du sein et de } \\
\text { douleurs aux mamelons } \\
\text { Diminution des douleurs aux } \\
\text { mamelons après l'intervention } \\
\text { (6,9 à 1,2) }\end{array}$ \\
\hline $\begin{array}{l}\text { Buryk et coll. } \\
\text { (26), } 2011\end{array}$ & $\begin{array}{c}\text { Essai aléatoire } \\
\text { et contrôlé à } \\
\text { simple insu }\end{array}$ & $\begin{array}{l}\text { Nourrissons } \\
\text { ayant des } \\
\text { problèmes } \\
\text { d'allaitement } \\
\text { et une } \\
\text { importante } \\
\text { ankyloglossie } \\
\text { (répartition } \\
\text { aléatoire à un } \\
\text { âge moyen de } \\
6 \text { jours) }\end{array}$ & $\begin{array}{l}\text { Score de la fonction } \\
\text { de HATLFF } \\
\text { supérieur à } 11 \text { sur } \\
14 \text { accompagné } \\
\text { de l'échec de } \\
\text { gestion de la } \\
\text { lactation ou d'un } \\
\text { score d'apparence } \\
\text { inférieur à } 8 \text { sur } 10\end{array}$ & $\begin{array}{l}\text { Affectés au hasard } \\
\text { entre une } \\
\text { frénotomie } \\
\text { (30 nourrissons) } \\
\text { et une } \\
\text { intervention } \\
\text { placebo } \\
\text { ( } 28 \text { nourrissons) } \\
\end{array}$ & $\begin{array}{l}\text { Allaitement évalué avant et après } \\
\text { l'intervention selon le } \\
\text { questionnaire court de McGill } \\
\text { sur la douleur aux mamelons } \\
\text { (SF-MPQ)(27) et l'IBFAT à } \\
2 \text { semaines et aux suivis } \\
\text { réguliers } \\
\text { Résultats secondaires de l'effet de } \\
\text { la frénotomie sur la durée de } \\
\text { l'allaitement }\end{array}$ & $\begin{array}{l}\text { Les } 2 \text { groupes présentaient une } \\
\text { diminution statistiquement } \\
\text { significative des scores de douleur } \\
\text { aux mamelons après l'intervention } \\
\text { Le score d'IBFAT ne différait pas de } \\
\text { manière significative entre le } \\
\text { groupe ayant subi une frénotomie } \\
\text { et le groupe témoin après } \\
\text { l'intervention } \\
27 \text { des } 28 \text { nourrissons du groupe } \\
\text { témoin ont subi une frénotomie au } \\
\text { suivi de } 2 \text { semaines ou auparavant } \\
\text { Aucune différence dans la durée de } \\
\text { l'allaitement } \\
\text { Compte rendu subjectif par la mère } \\
\text { d'une amélioration de la prise du } \\
\text { sein après la frénotomie }\end{array}$ \\
\hline
\end{tabular}

Si on constate une association entre une ankyloglossie marquée et de graves problèmes d'allaitement et qu'une intervention chirurgicale est jugée nécessaire, un clinicien habitué à l'exécuter doit se charger de la frénotomie, après une analgésie convenable.

Il est recommandé de consulter un professionnel de la santé qui possède des compétences en allaitement avant d'orienter le nourrisson vers une frénotomie.

\section{RECOMMANDATIONS}

Des critères clairs s'imposent pour diagnostiquer l'ankyloglossie, de même que les caractéristiques des nourrissons chez qui une frénotomie contribuerait à améliorer la tétée. Sur le plan du pronostic, il est essentiel de déterminer les caractéristiques de l'ankyloglossie qui pourraient aider le clinicien à sélectionner les enfants les plus susceptibles de tirer profit de la frénotomie. De plus, sur le plan de la méthodologie, les futures études devraient inclure un échantillon clinique plus important et éviter le croisement d'un groupe d'étude à l'autre.

Il est important d'écarter les autres anomalies buccales qui risquent de provoquer des problèmes d'allaitement. Ainsi, il faut effectuer un examen intraoral complet du nouveau-né, qui inclut l'inspection et l'évaluation de la fonction de la langue, surtout en présence de problèmes d'allaitement. Il faut ensuite poser des questions à la mère sur l'allaitement (prise du sein, douleurs aux mamelons, inconfort) et observer la tétée. En présence de problèmes démontrés, on peut envisager d'orienter la mère et le nourrisson vers un dispensateur de soins possédant des compétences en soutien à l'allaitement.

\section{RÉFÉRENCES}

1. Messner AH, Lalakea ML. Ankyloglossia: Controversies in management. Int J Pediatr Otorhinolaryngol 2000;54(2-3):123-31.

2. Wright JE. Tongue-tie. J Paediatr Child Health 1995;31(4):276-8.

3. Griffiths DM. Do tongue ties affect breastfeeding? J Hum Lact 2004;20(4):409-14.

4. Kupietzky A and Botzer E. Ankyloglossia in the infant and young child: Clinical suggestions for diagnosis and management. Pediatr Dent 2005;27(1):40-6.

5. Rowan-Legg A; Société canadienne de pédiatrie, comité de la pédiatrie communautaire. L'ankyloglossie et l'allaitement. Paediatr Child Health 2011;16(4):222.

6. Hogan M, Westcott C, Griffiths M. Randomized, controlled trial of division of tongue-tie in infants with feeding problems. J Paediatr Child Health 2005;41(5-6):246-50.

7. Ricke LA, Baker NJ, Madlon-Kay DJ, DeFor TA. Newborn tongue-tie: Prevalence and effect on breast-feeding. J Am Board Fam Pract 2005;18(1):1-7.

8. Ballard JL, Auer CE, Khoury JC. Ankyloglossia: Assessment, incidence, and effect of frenuloplasty on the breastfeeding dyad. Pediatrics 2002;110(5):e63.

9. Messner AH, Lalakea ML, Aby J, Macmahon J, Bair E. Ankyloglossia: Incidence and associated feeding difficulties. Arch Otolaryngol Head Neck Surg 2000;126(1):36-9.

10. O'Callahan C, Macary S, Clemente S. The effects of office-based frenotomy for anterior and posterior ankyloglossia on breastfeeding. Int J Pediatr Otorhinolaryngol 2013;77(5):827-32.

11. Hazelbaker AK. The Assessment Tool for Lingual Frenulum Function (ATLFF): Use in a lactation consultant's private practice. Pasadena: Pacific Oaks College, 1993.

12. Amir LH, James JP, Donath SM. Reliability of the Hazelbaker Assessment Tool for Lingual Frenulum Function. Int Breastfeed J 2006;1(1):3-8. 
13. Segal LM, Stephenson R, Dawes M, Feldman P. Prevalence, diagnosis, and treatment of ankyloglossia: Methodologic review. Can Fam Physician 2007;53(6):1027-33.

14. Notestine GE. The importance of the identification of ankyloglossia (short lingual frenulum) as a cause of breastfeeding problems. J Hum Lact 1990;6(3):113-5.

15. Berg KL. Tongue-tie (ankyloglossia) and breastfeeding: A review. J Hum Lact 1990;6(3):109-12.

16. Geddes DT, Kent JC, McClellan HL, Garbin CP, Chadwick LM, Hartmann PE. Sucking characteristics of successfully breastfeeding infants with ankyloglossia: A case series. Acta Paediatr 2010;99(2):301-3.

17. Garbin CP, Sakalidis VS, Chadwick LM, Whan E, Hartmann PE, Geddes DT. Evidence of improved milk intake after frenotomy: A case report. Pediatrics 2013;132(5):e1413-17.

18. Wight NE. Management of common breastfeeding issues. Pediatr Clin North Am 2001;48(2):321-44.

19. Geddes DT, Langton DB, Gollow I, Jacobs LA, Hartmann PE, Simmer K. Frenulotomy for breastfeeding infants with ankyloglossia: Effect on milk removal and sucking mechanism as imaged by ultrasound. Pediatrics 2008;122(1):e188-94.

20. Dollberg S, Botzer E, Grunis E, Mimouni FB.Immediate nipple pain relief after frenotomy in breast-fed infants with ankyloglossia: A randomized, prospective study. J Pediatr Surg 2006;41(9):1598-600.

21. Jensen D, Wallace S, Kelsay P. LATCH: A breastfeeding charting system and documentation tool. J Obstet Gynecol Neonatal Nurs 1994;23(1):27-32.

22. Berry J, Griffiths M, Westcott C. A double-blind, randomized, controlled trial of tongue-tie division and its immediate effect on breastfeeding. Breastfeed Med 2012;7(3):189-93.

23. Matthews MK. Developing an instrument to assess infant breastfeeding behaviour in the early neonatal period. Midwifery 198814(4):154-65.
24. Emond A, Ingram J, Johnson D et coll. Randomised controlled trial of early frenotomy in breastfed infants with mild-moderate tongue-tie. Arch Dis Child Fetal Neonatal Ed 2014;99(3):F189-95.

25. Dennis CL. The breastfeeding self-efficacy scale: Psychometric assessment of the short form. J Obstet Gynecol Neonatal Nurs 2003;32(6):734-44.

26. Buryk M, Bloom D, Shope T. Efficacy of neonatal release of ankyloglossia: A randomized trial. Pediatrics 2011;128(2):280-8.

27. Melzack R. The short-form McGill Pain Questionnaire. Pain 1987;30(2):191-7.

28. Ngerncham S, Laohapensang M, Wongvisutdhi T et coll. Lingual frenulum and effect on breastfeeding in Thai newborn infants. Paediatr Int Child Health 2013;33(2):86-90.

29. Post E, Daamen J, Balemans W. Snipping of a "tongue tie" in neonates with ankyloglossia and breastfeeding problems: Outcomes and complications. Arch Dis Child 2012:97(Suppl 2):A486.

30. Kumar M, Kalke E. Tongue-tie, breastfeeding difficulties and the role of frenotomy. Acta Paediatr 2012;101(7):687-9.

31. Sethi N, Smith D, Kortequee S, Ward VM, Clarke S. Benefits of frenulotomy in infants with ankyloglossia. Int J Pediatr Otorhinolaryngol 2013;77(5):762-5.

32. Dollberg S, Marom R, Botzer E. Lingual frenotomy for breastfeeding difficulties: A prospective follow-up study. Breastfeed Med 2014;9(6):286-9.

33. Ovental A, Marom R, Botzer E, Batscha N, Dollberg S. Using topical benzocaine before lingual frenotomy did not reduce crying and should be discouraged. Acta Pediatr 2014;103(7):780-2.

34. Catlin FI. Tongue-tie. Arch Otolaryngol 1971;94(6):548-57.

\section{COMITÉ DE LA PÉDIATRIE COMMUNAUTAIRE DE LA SCP}

Membres : Carl Cummings MD (président); Sarah Gander MD; Ruth B Grimes MD; Barbara Grueger MD (membre sortante); Larry B Pancer MD; Anne Rowan-Legg MD; Ellen P Wood MD (représentante du conseil)

Représentant : Fabian P Gorodzinsky MD, section de la pédiatrie générale de la SCP

Auteure principale : Anne Rowan-Legg MD

Les recommandations contenues dans le présent document ne sont pas indicatrices d'un seul mode de traitement ou d'intervention. Des variations peuvent convenir, compte tenu de la situation. Tous les documents de principes et les points de pratique de la Société canadienne de pédiatrie sont régulièrement révisés. Les documents de principes retirés sont supprimés du site Web. Consultez la zone Documents de principes du site Web de la SCP (www.cps.ca) pour en obtenir la version complète à jour. 